\title{
Psoriasis and vitiligo are close relatives
}

This article was published in the following Dove Press journal:

Clinical, Cosmetic and Investigational Dermatology

29 August 2017

Number of times this article has been viewed

\section{Khalifa E Sharquie' \\ Husam Ali Salman' \\ Aseel K Yaseen ${ }^{2}$}

'Department of Dermatology and Venereology, College of Medicine, University of Baghdad, Baghdad, Iraq, ${ }^{2}$ Department of Dermatology and Venereology, Baghdad Teaching Hospital, Baghdad, Iraq
Correspondence: Husam Ali Salman Department of Dermatology and Venereology, College of Medicine, University of Baghdad, Medical Collection Office, PO Box 61211, Baghdad 12114, Iraq

Tel +9647709243027

Email husjob2000@yahoo.com
Background: Both vitiligo and psoriasis are chronic inflammatory autoimmune diseases with genetic elements.

Objective: To estimate the frequencies of psoriasis in vitiligo patients and vice versa and to compare them with healthy controls.

Patients and methods: A total of 1000 subjects were included, 250 of them had vitiligo, 250 had psoriasis, and 500 were healthy controls. Measurement of the frequencies of vitiligo in psoriatic patients and psoriasis in vitiligo patients was carried out. Thereafter the frequencies of both diseases were assessed in healthy controls. The frequency of vitiligo among psoriatic patients was compared with that of vitiligo in healthy controls. A similar comparison was done between the frequency of psoriasis among vitiligo patients with that in healthy controls. Other comparisons were performed between the frequency of family history of psoriasis among vitiligo patients with that in healthy controls and between the frequency of family history of vitiligo in psoriatic patients with that in healthy controls.

Results: The frequency of psoriasis among vitiligo patients was $15(6 \%)$ and among healthy controls was $2(0.4 \%)$; there is a statistically significant difference $(P=0.001)$. The frequency of vitiligo among psoriatic patients was $5(2 \%)$ and among healthy controls was $3(0.6 \%)$; no statistically significant difference was found $(P=0.16)$. The family history of psoriasis among vitiligo patients was $23(9.2 \%)$ and among healthy controls was $20(4 \%)$; there is a significant association $(P=0.043)$. The family history of vitiligo among psoriatic patients was $24(9.6 \%)$ and among healthy controls was $40(8 \%)$; the difference is statistically significant $(P=0.042)$.

Conclusion: The present work has confirmed the close relationship between vitiligo and psoriasis. Keywords: psoriasis, vitiligo, overlap, relationship

\section{Introduction}

Psoriasis is a common, chronic, disfiguring, inflammatory, and proliferative condition of the skin, in which both genetic and environmental influences have a critical role. ${ }^{1}$

Skin diseases associated with psoriasis include acquired bullous disorders, most notably, bullous pemphigoid ${ }^{2}$ and vitiligo ${ }^{3}$ with psoriasis sometimes reported to be confined to vitiliginous areas. Crohn's disease, ulcerative colitis, and psoriasis share an important association with sacroiliitis and HLA-B27 positivity. ${ }^{4}$

Vitiligo is a common autoimmune inflammatory disease where there is absence, deficiency, or dysfunction of melanocytes. ${ }^{5-7}$ Vitiligo is not infrequently associated with a number of clinical disorders that are considered to be autoimmune in their etiology. ${ }^{8,9}$ 
However, no study was carried out extensively searching the link between psoriasis and vitiligo. Accordingly, the aim of the present work is to support and confirm the clinical observation of close relationship between vitiligo and psoriasis rather than just association.

\section{Patients and methods}

This was a case-control study, carried out at the department of dermatology and venereology, Baghdad Teaching Hospital, in the period from 1st of April 2014 to the end of March 2015. A total of 1000 subjects were included in this study, 250 of them had vitiligo, 250 had psoriasis, and the rest 500 were age- and sex-matched healthy controls attending the hospital for other nonmedical reasons.

All patients were interrogated and a full history was taken regarding the age, sex, duration of the disease, occupation, marital status, alcohol drinking and smoking, family, and past medical history with special emphasis on thyroid diseases, diabetes mellitus, alopecia areata (AA), inflammatory bowel disease, and rheumatoid arthritis (RA).

For each patient an examination of the lesions whether psoriasis, vitiligo, or both was carried out to assess the type, extent, site, and for the presence of overlapping lesions of psoriasis and vitiligo.

This study was approved by the ethical committee of the Iraqi board for medical specializations and the Scientific Committee in the Scientific Council of Dermatology and Venereology.

A written informed consent was obtained from each participant, or their parents or legal guardians for those under the age of 18 years, both for participation in the study and for the use of images in the paper.

Measurement of the frequency of vitiligo among psoriatic patients and vice versa was carried out. Thereafter the frequency of both diseases was assessed in healthy controls attending the hospital for other purposes as a control group.

The frequency of vitiligo among psoriatic patients was compared with that of vitiligo in healthy controls. A similar comparison was done between the frequency of psoriasis among vitiligo patients with that in healthy controls.

Other comparisons were performed between the frequency of family history of psoriasis among vitiligo patients with that in healthy control and between the frequency of family history of vitiligo in psoriatic patients with that in healthy control.

\section{Results}

A total of 1000 study participants were included in the present study. Their ages ranged from 14 to 68 years.
The mean age of vitiligo patients was $27.13 \pm 8.07$ with a male-to-female ratio of $0.6: 1$. The mean duration of vitiligo was $2.1 \pm 5.2$ years.

The mean age of psoriatic patients was $26.58 \pm 7.62$ with a male-to-female ratio of $0.56: 1$. The mean duration of psoriasis was $2.2 \pm 5.6$ years.

For the healthy controls the mean age was $28.02 \pm 8.48$ with a male-to-female ratio of 0.73:1.

The frequency of psoriasis among patients with vitiligo was $15(6 \%) ; 8$ (3.2\%) patients had psoriasis within vitiligo lesions (Figure 1) and 9 (3.6\%) outside vitiligo patches (two of them had both inside and outside lesions; Table 1).

The frequency of psoriasis among healthy control was 2 $(0.4 \%)$ versus $15(6 \%)$ among vitiligo patients. There was a statistically significant difference $(P$ value $<0.001$; Table 2$)$.

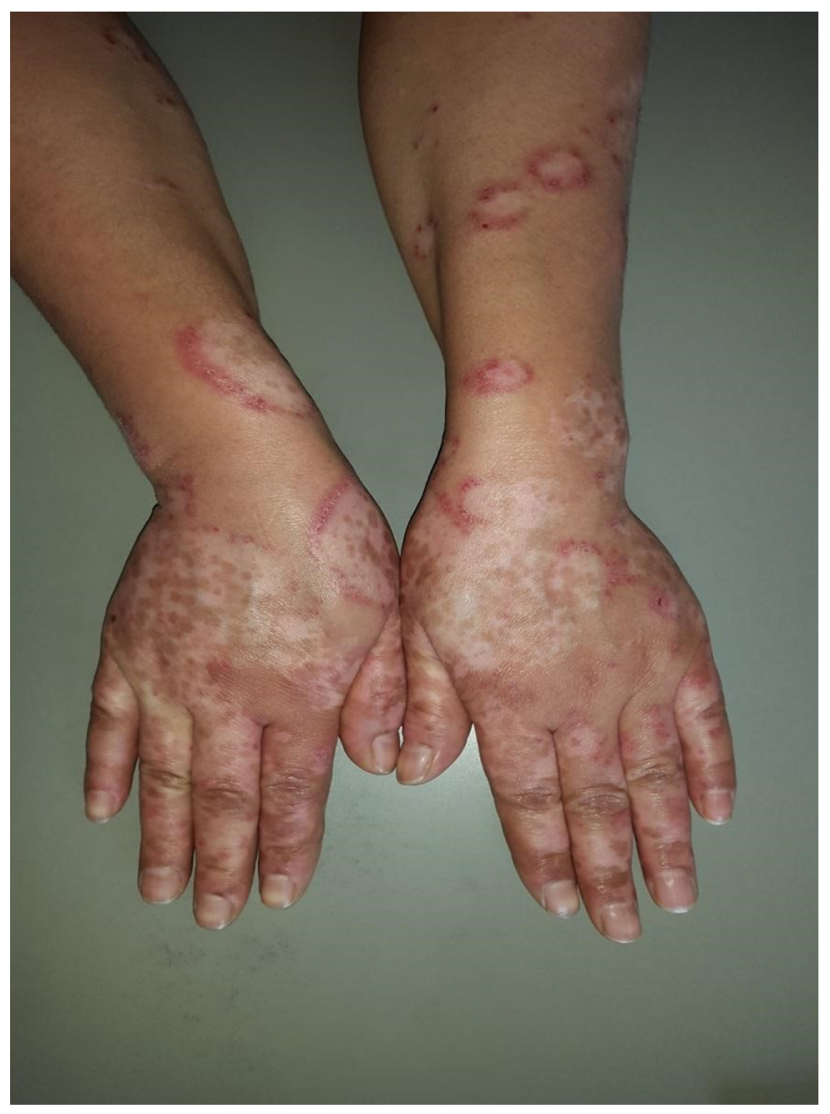

Figure I Psoriatic lesions superimposed on vitiligo patches and forming advancing border of vitiligo lesion.

Table I The frequency of psoriasis among vitiligo patients

\begin{tabular}{ll}
\hline Patients with vitiligo & N (\%) \\
\hline Total number of patients with psoriasis in vitiligo patients & I5 (6) \\
Psoriasis within vitiligo lesion & $8^{*}(3.2)$ \\
Total number of psoriasis out of lesion of vitiligo & $9 *(3.6)$ \\
Vitiligo patients without psoriasis & $235(94)$ \\
Total & $250(100)$ \\
\hline
\end{tabular}

Notes: *Two patients had psoriasis in and out of lesion of vitiligo. 
The frequency of vitiligo among psoriatic patients was 5 $(2 \%) ; 2(0.8 \%)$ within psoriatic lesions and $3(1.2 \%)$ outside (Table 3).

The frequency of vitiligo among healthy controls was 3 $(0.6 \%)$ versus $5(2 \%)$ and among psoriatic patients. There was no significant association (Table 4).The family history of vitiligo among vitiligo patients was positive in $100(40 \%)$ cases and for psoriasis among psoriasis patients was positive in $81(32.4 \%)$ cases.

The family history of psoriasis was more frequent among vitiligo patients than healthy control; $9.2 \%$ versus $4 \%$, respectively. The difference was statistically significant. $P$ value $<0.05$ (Table 5).

Table 2 The frequency of psoriasis in vitiligo patients and healthy controls

\begin{tabular}{|c|c|c|c|c|c|c|c|}
\hline \multirow[t]{2}{*}{ Psoriasis } & \multicolumn{2}{|c|}{ Vitiligo } & \multicolumn{2}{|c|}{ Healthy control } & \multirow[t]{2}{*}{$\chi^{2}$} & \multirow[t]{2}{*}{$d f$} & \multirow[t]{2}{*}{$P$-value } \\
\hline & $\mathbf{N}$ & $\%$ & $\mathbf{N}$ & $\%$ & & & \\
\hline Yes & 15 & 6.0 & 2 & 0.4 & 23.5 & 1 & $<0.001$ \\
\hline No & 235 & 94.0 & 498 & 99.6 & & & \\
\hline Total & 250 & 100.0 & 500 & 100.0 & & & \\
\hline
\end{tabular}

Table 3 The frequency of vitiligo among psoriatic patients

\begin{tabular}{ll}
\hline Patients with psoriasis & $\mathbf{N}(\%)$ \\
\hline $\begin{array}{l}\text { Total number of patients with vitiligo in psoriatic } \\
\text { patients }\end{array}$ & $5(2)$ \\
$\quad$ Vitiligo within lesion of psoriasis & $2(0.8)$ \\
$\quad$ Vitiligo out of lesion of psoriasis & $3(1.2)$ \\
Psoriatic patients without vitiligo & $245(98)$ \\
Total & $250(100.0)$ \\
\hline
\end{tabular}

Table 4 The frequency of vitiligo in patients with psoriasis and healthy controls

\begin{tabular}{|c|c|c|c|c|c|c|c|}
\hline \multirow[t]{2}{*}{ Vitiligo } & \multicolumn{2}{|c|}{ Psoriasis } & \multicolumn{2}{|c|}{ Healthy control } & \multirow[t]{2}{*}{$\chi^{2}$} & \multirow[t]{2}{*}{$d f$} & \multirow[t]{2}{*}{$P$-value } \\
\hline & $\overline{\mathbf{N}}$ & $\%$ & $\overline{\mathbf{N}}$ & $\%$ & & & \\
\hline Yes & 5 & 2.0 & 3 & 0.6 & 3.09 & 1 & 0.16 \\
\hline No & 245 & 98.0 & 497 & 99.4 & & & \\
\hline Total & 250 & 100.0 & 500 & 100.0 & & & \\
\hline
\end{tabular}

Table 5 Family history of vitiligo and psoriasis among vitiligo patients in comparison with control

\begin{tabular}{|c|c|c|c|c|c|c|c|}
\hline \multirow[t]{2}{*}{ Variable } & \multicolumn{2}{|c|}{ Vitiligo } & \multicolumn{2}{|c|}{ Healthy control } & \multirow[t]{2}{*}{$\chi^{2}$} & \multirow[t]{2}{*}{$d f$} & \multirow[t]{2}{*}{$P$-value } \\
\hline & $\overline{\mathbf{N}}$ & $\%$ & $\overline{\mathbf{N}}$ & $\%$ & & & \\
\hline \multicolumn{5}{|c|}{ Family history of vitiligo } & 87.9 & 1 & $<0.001$ \\
\hline No & 150 & 60.0 & 460 & 92.0 & & & \\
\hline Yes & 10 & 40.0 & 40 & 8.0 & & & \\
\hline Total & 250 & 100.0 & 500 & 100.0 & & & \\
\hline \multicolumn{5}{|c|}{ Family history of psoriasis } & 3.42 & 1 & $<0.05$ \\
\hline No & 227 & 90.8 & 480 & 96.0 & & & \\
\hline Yes & 23 & 9.2 & 20 & 4.0 & & & \\
\hline Total & 250 & 100.0 & 500 & 100.0 & & & \\
\hline
\end{tabular}

The family history of vitiligo was more frequent in psoriatic patients than healthy control; $9.6 \%$ versus $8 \%$, respectively. The difference was statistically significant. $P$ value $<0.05$ (Table 6).

Psoriasis lesion healed with hypopigmentation and leukoderma that could simulate vitiligo patches (Figures 2 and 3 ) in $20(8 \%)$ patients, while only one $(0.4 \%)$ patient was healed with hyperpigmentation and the majority, 229 (91.6\%), of psoriatic lesions were either not healed or healed without any residual pigmentary changes.

Close analysis of results had shown that patients with vitiligo had a higher chance to develop psoriasis while

Table 6 Family history of psoriasis and vitiligo among psoriatic patients in comparison with that of control

\begin{tabular}{|c|c|c|c|c|c|c|c|}
\hline \multirow[t]{2}{*}{ Variable } & \multicolumn{2}{|c|}{ Psoriasis } & \multicolumn{2}{|c|}{ Healthy control } & \multirow[t]{2}{*}{$\chi^{2}$} & \multirow[t]{2}{*}{$d f$} & \multirow[t]{2}{*}{$P$-value } \\
\hline & $\overline{\mathbf{N}}$ & (\%) & $\overline{\mathbf{N}}$ & (\%) & & & \\
\hline \multicolumn{8}{|c|}{ Family history of psoriasis } \\
\hline No & 169 & 67.6 & 480 & 96.0 & 70.56 & 1 & $<0.001$ \\
\hline Yes & 81 & 32.4 & 20 & 4 & & & \\
\hline Total & 250 & 100 & 500 & 100.0 & & & \\
\hline \multicolumn{8}{|c|}{ Family history of vitiligo } \\
\hline No & 226 & 90.4 & 460 & 92.0 & 4.325 & 1 & $<0.05$ \\
\hline Yes & 24 & 9.6 & 40 & 8 & & & \\
\hline Total & 250 & 100 & 500 & 100.0 & & & \\
\hline
\end{tabular}

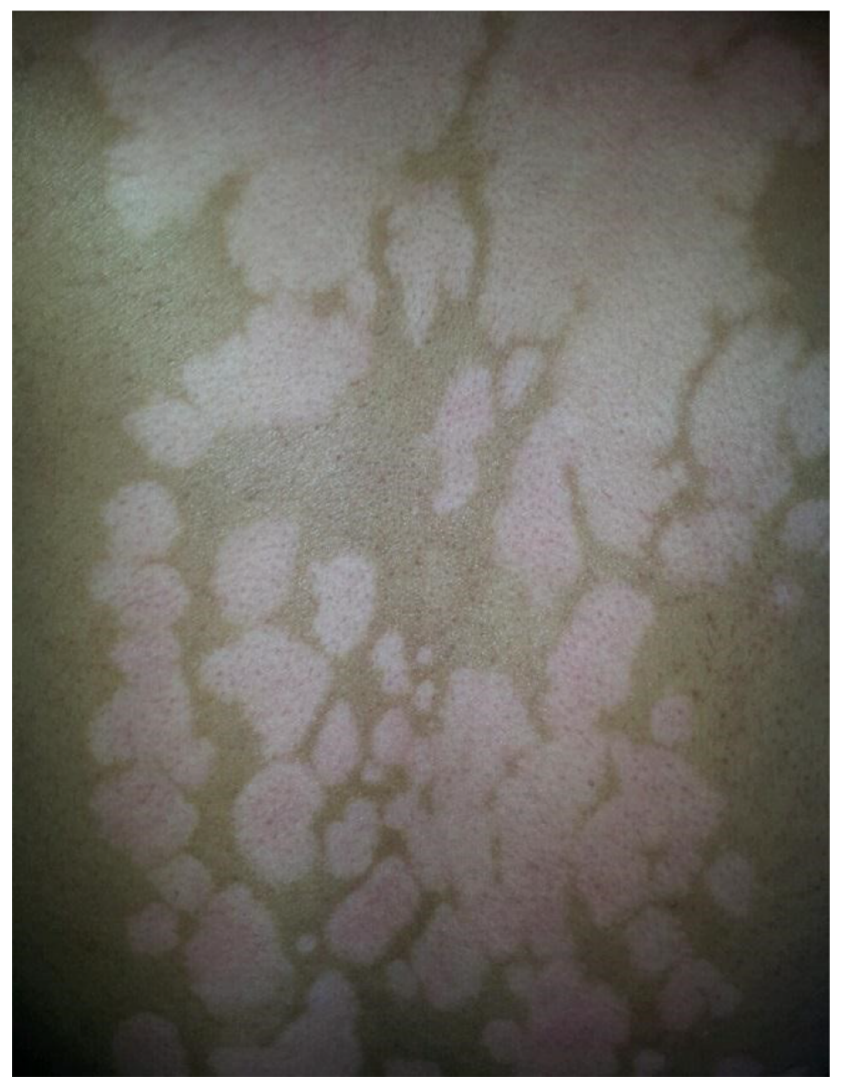

Figure 2 Postinflammatory hypopigmentation after resolution of psoriasis. 


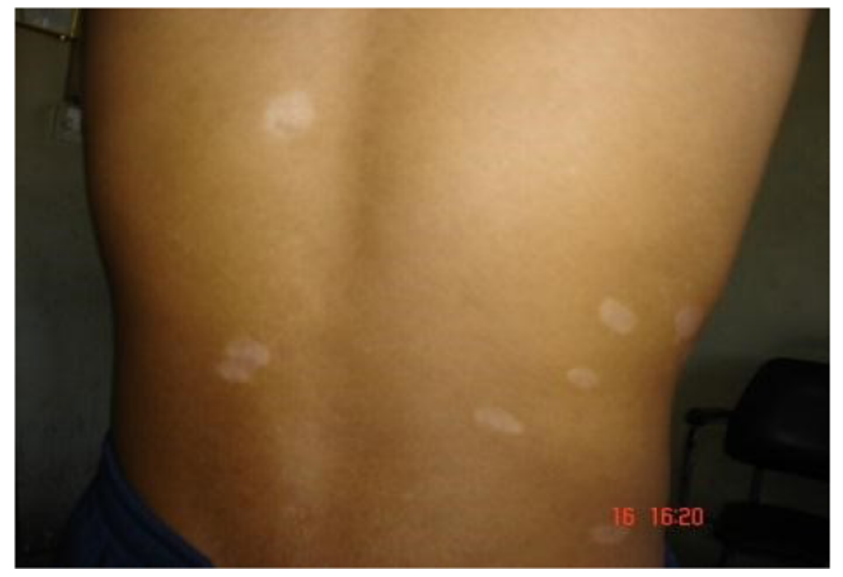

Figure 3 Leukoderma or vitiligo following healing of psoriasis.

patients with psoriasis had a lower chance to develop vitiligo as frequency of psoriasis among vitiligo patients was $6 \%$ while the frequency of vitiligo among psoriasis was $2 \%$ only.

\section{Discussion}

Both vitiligo and psoriasis are inflammatory autoimmune diseases where Th1 plays a role in its pathogenesis ${ }^{4,10}$ and have genetic elements. ${ }^{4,8,9}$

A study in China found that rs. 9468925 in HLA-C/ HLA-B is associated with both psoriasis and vitiligo, providing first important evidence that two major skin diseases share a common genetic locus in the major histocompatibility complex (MHC) and a basic for elucidating the molecular mechanism of skin disorders. ${ }^{11}$ Between $30 \%$ and $40 \%$ of vitiligo patients have a positive family history of vitiligo, ${ }^{9}$ and a positive family history had been reported by $35 \%-90 \%$ of patients with psoriasis. ${ }^{4}$

These results were similarly observed in the present study as family history of vitiligo was $40 \%$ and family history of psoriasis was $37.2 \%$. Both have positive Kobner phenomena, ${ }^{8-12}$ and most of the psoriatic lesions heal with postinflammatory hypopigmentation. ${ }^{13}$

Most of the psoriatic lesions heal with postinflammatory hypopigmentation that could not even be easily differentiated from vitiligo as observed in the present work. Also in many cases, it was difficult to say which disease started first, vitiligo or psoriasis, or occurred both at the same time.

There are many reports showing an association with psoriasis in the same patient. The first case of psoriasis accompanied with vitiligo was reported by Selenyi. ${ }^{14}$ Park reported a case of coexisting vitiligo and psoriasis in the same individual at different sites and he reviewed the relevant literature. ${ }^{15}$ From a review of another literature, 22 (2.67\%) out of 821 Egyptian patients and 3 (4.83\%) of 62 British patients with vitiligo also had psoriasis. ${ }^{16,17}$ In Italy, there was an interesting retrospective study of 712 patients with vitiligo, and 21 (3\%) patients had associated psoriasis. ${ }^{3}$ Sandhu et al reported through a retrospective study that among the 4700 patients with psoriasis, $38(0.8 \%)$ patients had associated vitiligo. ${ }^{18}$ While in the present work $6 \%$ of patients with vitiligo had psoriasis and $2 \%$ of patients with psoriasis had vitiligo, which was statistically significant when compared with the control. Close analysis of results, in the present work, had shown that patients with vitiligo had a higher chance to develop psoriasis while patients with psoriasis has lower chance to develop vitiligo as frequency of psoriasis among vitiligo patients was $6 \%$ while the frequency of vitiligo among psoriasis was $2 \%$ only. Hence, all previous studies and the results of the present work had confirmed the close link between vitiligo and psoriasis but not coincidental association.

The documents in favor of close link are as follows: genetic and immunological factors are present in both diseases, and the two major skin diseases share a common genetic locus in the MHC and a basic for elucidating the molecular mechanism of skin disorders, Th1 cytokines play a role in the etiopathogenesis of both diseases. Also, $6 \%$ of patients with vitiligo develop psoriasis; healing of psoriatic lesions often leaves hypopigmentation that could simulate vitiligo and leaves no hyperpigmentation. In addition, psoriasis appears in many patients only in vitiligenous areas.

\section{Disclosure}

The authors report no conflicts of interest in this work.

\section{References}

1. Griffiths CEM, Barker JNWN. Psoriasis. In: Burns T, Breathnach S, Cox N, Griffiths C, editors. Rook's Textbook of Dermatology. 8th ed. Singapore: Wiley Blackwell Publishing Company; 2010;20:871-930.

2. Wilczek A, Sticherling M. Concomitant psoriasis and bullous pemphigoid: coincidence or pathogenic relationship? Int $J$ Dermatol. 2006;45:1353-1357.

3. Percivalle S, Piccinno R, Caccialanza M. Concurrence of vitiligo and psoriasis: a simple coincidence? Clin Exp Dermatol. 2009;34:90-91.

4. Van de Kerkhof PCM, Schalkwijk J. Psoriasis. In: Bolognia JL, Jorizzo JL, Rapini RP, editors. Dermatology. 2nd ed. Barcelona, Spain: Mosby Elsevier; 2010;9:115-135.

5. Sharquie KE. The Histology and Immunopathology of Vitiligo $[\mathrm{PhD}$ thesis]. England: University of Sheffield; 1982.

6. Sharquie KE. Stages of depigmentation in vitiligo. Iraqi Medical J. 1988;36:47-50.

7. Sharquie KE, Ahmed S, Naji A, Al Azawi H. Inflammatary changes in vitiligo. Am J Dermatopathol. 2004;26(2):108-112.

8. Lapeere H, Bonne B, Schepper SD, et al. Hypomelanoses and Hypermelanoses. In: Wolf K, Goldsmith LA, Katz SI, Gilchrest BA, Paller AS, Leffel DJ, editors. Fitzpatrick's Dermatology in General Medicine, 7th ed. New York, NY: McGraw-Hills; 2008:839-847.

9. Bleehen. SS, Ebling FJG. Disorders of skin color. In: Rook A. Wilkinson DS, Ebling FJG, Champion RH, Burton JL, editors. Textbook of Dermatology. 8th ed. Oxford: Blackwell Scientific Publication; 2010:58.46-48. 
10. Lee DJ, Modlin RL. Breaking tolerance-another piece added to the vitiligo puzzle. J Invest Dermatol. 2005;124(1):xiiiv.

11. Zhu KJ, Lv YM, Yin XY, et al. Psoriasis regression analysis of MHC loci identifies shared genetic variants with vitiligo. PLoS One. 2011;6(11):e23089.

12. Weiss G, Shemer A, Trau H. The Koebner phenomenon: review of the literature. J Eur Acad Dermatol Venereol. 2002;16(3):241-248.

13. Ortonne JP. Vitiligo and other disorders of hypopigmentation. Dermatology. 2008;1:947-973.

14. Selenyi A. Vitiligo and Psoriasis on the same side with syringomyelia. Borgyogy Venerol Sz. 1955;9:94-96.
15. Park JM, Kim JH, Bae BG, Park YK. A case of concurrent vitiligo and psoriasis. Ann Dermatol. 2009;21(3):330-333.

16. El-Mofty AM. Vitiligo. A symptom complex. Int J Dermatol. 1980;19(5): 237-244.

17. Bor S, Feiwel M, Chanarin I. Vitiligo and its aetiological relationship to organ-specific autoimmune disease. Br J Dermatol. 1969;81: $83-88$.

18. Sandhu K, Kaur I, Kumar B. psoriasis and vitiligo. JAm Acad Dermatol. 2004;51:149-150.
Clinical, Cosmetic and Investigational Dermatology

\section{Publish your work in this journal}

Clinical, Cosmetic and Investigational Dermatology is an international, peer-reviewed, open access, online journal that focuses on the latest clinical and experimental research in all aspects of skin disease and cosmetic interventions. This journal is included on PubMed. The manuscript management system is completely online

\section{Dovepress}

and includes a very quick and fair peer-review system, which is all easy to use. Visit http://www.dovepress.com/testimonials.php to read real quotes from published authors

Submit your manuscript here: https://www.dovepress.com/clinical-cosmetic-and-investigational-dermatology-journal 